\title{
A remark on ill-posedness
}

Haibo Yang ${ }^{1}$, Qixiang Yang ${ }^{2}$, and Huo Xiong $\mathrm{Wu}^{3}$

${ }^{1}$ Hubei University

${ }^{2}$ Wuhan University

${ }^{3}$ Xiamen University

March 20, 2021

\begin{abstract}
In this paper, we construct an example to show that wellposedness and norm inflation are compatible.
\end{abstract}

\section{Hosted file}

remark-ill.pdf available at https://authorea.com/users/402705/articles/514486-a-remark-onill-posedness 